ORIGINAL

\title{
Differences In High-Intensity Signal Volume Between Arterial Spin Labeling And Contrast-Enhanced T1-Weighted Imaging May Be Useful For Differentiating Glioblastoma From Brain Metastasis
}

\author{
Mungunbagana Ganbold ${ }^{1}, \mathrm{MD}$; Masafumi Harada${ }^{1}, \mathrm{MD}, \mathrm{PhD}$; Delgerdalai Khashbat ${ }^{1}, \mathrm{MD}, \mathrm{PhD}$; \\ Takashi Abe ${ }^{1}, \mathrm{MD}, \mathrm{PhD}$; Teruyoshi Kageji², MD, PhD ; and Shinji Nagahiro², MD, PhD \\ ${ }^{1}$ Department of Radiology and Radiation Oncology, ${ }^{2}$ Department of Neurosurgery, Institute of Biomedical Sciences, Tokushima University \\ Graduate School
}

\begin{abstract}
Purpose : To determine whether differences in tumor volume between arterial spin labeling (ASL) and contrast-enhanced T1 - weighted MR images (CE+T1WI) can help differentiate glioblastoma (GBM) from brain metastasis. Materials and methods : Patients with a diagnosis of GBM $(n=25)$ or brain metastasis $(n=13)$ were examined by both conventional and ASL MR imaging. Volumes of interest with high signal intensity on ASL and CE+T1WI were defined using three dimensional analysis software. Tumor volume difference (ASL-CE) and tumor volume ratio (ASL/CE) were obtained. Absolute maximal tumor blood flow (TBF) and TBF ratio (normalized to white matter) were also measured. The Mann-Whitney $U$ test and receiver operating characteristic curve analysis were performed to compare measurements between the tumor groups. Results : Both tumor volume difference and tumor volume ratio were significantly higher in GBM than in metastasis. Both TBF and TBF ratio were higher for GBM than for metastasis, but the differences were not significant. Conclusion : The difference in tumor volume as measured by ASL high signal intensity and CE+T1WI might be useful for differentiating GBM from metastasis, whereas ASL-derived TBF is insufficient. J. Med. Invest. 64 : 58-63, February, 2017
\end{abstract}

Keywords : arterial spin labeling, perfusion, volumetric analysis, glioblastoma, metastasis

\section{INTRODUCTION}

Glioblastoma (GBM) and brain metastasis are the most common brain neoplasms in adulthood, accounting for about half of all cases $(1,2)$. It is critical to distinguish GBM from metastasis for prognosis and optimal treatment. However, differential diagnosis is generally a challenge using conventional magnetic resonance imaging (MRI) because both of these neoplasms show similar imaging manifestations, like a solid contrast enhancement with peritumoral edema and a mass effect.

Perfusion MRI is helpful for the assessment of brain tumors by defining the degree of microvascular proliferation. Studies using dynamic susceptibility contrast-enhanced (DSC) MRI, the most prevalent perfusion technique, reported a relatively higher value for GBM compared with metastasis. This difference stems from the peritumoral perfusion deficit caused by edema surrounding metastatic tumors (3-5). However, the presence of tumoral edema and the difference in perfusion are still insufficient for differentiation due to a high likelihood of elevated perfusion value in metastasis (6) and tumoral edema in primary gliomas (7).

As an alternative to DSC MRI, arterial spin labeling (ASL) perfusion imaging has proven to be reliable for evaluating cerebral blood circulation. In contrast to standard perfusion imaging with exogenous contrast agents, ASL uses arterial blood water as an endogenous tracer to quantify cerebral blood flow (CBF) (8).

Received for publication October 5, 2016 ; accepted October 23, 2016.

Address correspondence and reprint requests to Mungunbagana Ganbold, MD ; Department of Radiology and Radiation Oncology, Institute of Biomedical Sciences, Tokushima University Graduate School 3-18-15 Kuramoto-cho, Tokushima City, Tokushima 770-8509, Japan and Fax : $+81-88-633-7174$.
Estimation of CBF parameters derived from ASL are used in clinical practice to assess various neurological disorders, including tumors, stroke, and epilepsy $(9,10)$. In addition to CBF estimation, several groups have reported that a high-intensity signal abnormality on ASL images could aid in the diagnosis of cerebrovascular malformations $(11,12)$. Furthermore, a recent study conducted at our institution demonstrated that high signal intensity on ASL may be used to distinguish histologically distinct tumors (13). In that investigation, a mismatch in the high-intensity signal area between ASL and contrast-enhanced imaging was found for primary gliomas and malignant lymphomas compared to metastasis.

While conventional MRI manifestations are comparable, mechanisms of tumor angiogenesis differ markedly between GBM and metastasis. GBM is a highly vascular tumor, and microvessels always infiltrate into surrounding brain tissue $(4,5,14)$. In contrast, there is a lack of angiogenic infiltration into peritumoral tissue in metastasis (15). ASL is a very sensitive modality for detecting hypervascularity, which appears as high-intensity signals (10). On the other hand, the main source of estimation errors in ASL imaging is often related to hypoperfused lesions with arterial transit delay (9).

Various volumetric parameters derived from the high-intensity signals on traditional MRI sequences, such CE+T1WI, FLAIR, and T2WI, are used for brain tumor evaluation, especially volumetric changes in contrast enhancement (16-18). It has been reported that three-dimensional (3D) volumetry maybe more accurate for defining tumor volume than estimates based on $2 \mathrm{D}$ axial slices (19).

In this study, we measured the volumes of the ASL high-intensity signals from GBM and metastasis and compared them with those of CE+T1WI signals, allowing sensitive differentiation of these 
tumor types using the subtracted high-intensity volume (ASL minus $\mathrm{CE}+\mathrm{T} 1 \mathrm{WI}$ ) and ratio (ASL to $\mathrm{CE}+\mathrm{T} 1 \mathrm{WI})$. We have also compared $\mathrm{CBF}$ variables estimated by ASL between the tumor groups.

\section{MATERIALS AND METHOD}

This study was approved by the ethics committee of our institution and informed consent was obtained from all patients before the examinations.

We selected prospective patients with GBM or metastasis who had undergone MRI examinations at Tokushima University Hospital between 2010 and 2015. All patients had been evaluated by ASL and other MRI sequences using the same MRI machine prior to treatment. Of 41 patients considered for this study, 1 case with metastasis was excluded due to a visible artifact on ASL images and $2 \mathrm{GBM}$ cases were excluded due to lack of contrast enhancement on $\mathrm{CE}+\mathrm{T} 1 \mathrm{WI}$.

Finally, 38 patients (23 men, 15 women ; age range $43-87$ years ; mean age, 65.26 years) with a diagnosis of GBM or metastasis were enrolled. All 25 patients with GBM were diagnosed by histopathological examination at surgical resection or biopsy, and histopathological results were used as the reference based on World Health Organization criteria (20). Of the 13 patients with metastasis, 11 were diagnosed by histopathological examination and 2 were diagnosed by clinical findings (21). The primary metastatic tumor was lung carcinoma in 5 patients, breast carcinoma in 3 , colon carcinoma in 3 , urothelial carcinoma in 1 , and ovarian carcinoma in 1 .

\section{MRI PROTOCOLS}

MRI was performed on a 3-T scanner (Discovery 750, GE Healthcare, Milwaukee, WI) using a standard 8-channel head coil. All MRI examinations included ASL, CE+T1WI, T2WI, FLAIR, T1WI, and DWI.

$\mathrm{CE}+\mathrm{T} 1 \mathrm{WI}$ with 3D fast spoiled gradient echo (FSPGR) images were acquired as following parameter. Four minutes after injection of $0.1 \mathrm{mmol} / \mathrm{kg}$ body weight a gadolinium-containing contrast agent (gadopentetate dimeglumine Gd-DTPA, Magnevist, Bayer HealthCare, Berlin, Germany) with a power injector (Medrad Inc, Indianola, PA, USA) at $2.5 \mathrm{ml} / \mathrm{s}$ followed with normal saline $(20 \mathrm{ml})$ lock-flush at the same rate. Scan parameters for 3D FSPGR were : TR $10.4 \mathrm{~ms}$, TE $4.4 \mathrm{~ms}$, bandwidth $31.25 \mathrm{kHz}$, slice thickness 1.2 $\mathrm{mm}$, matrix $384 \times 256$, flip angle $15^{\circ}$, NEX one, field of view (FOV) $24 \times 24 \mathrm{~cm}$, acceleration factor $2 \times 2$, number of slices 160 , and acquisition time of $3: 34 \mathrm{~min}$.

ASL was obtained with pseudo-continuous labeling, background suppression, and a stack of spiral 3D fast-spin echo imaging sequences (22). Acquisition parameters were as follows : 512 sampling points on 8 spirals, field of view (FOV) $24 \mathrm{~cm}$, TR $4632 \mathrm{~ms}$, TE $10.5 \mathrm{~ms}$, reconstructed matrix size $64 \times 64$, number of excitations 2, post-labeling delay $1525 \mathrm{~ms}$, slice thickness $4 \mathrm{~mm}$, number of slices 36 , and total acquisition time $3: 15 \mathrm{~min}$.

\section{IMAGING DATA ANALYSIS}

We measured the tumor volumes by contrast enhancement on $\mathrm{CE}+\mathrm{T} 1 \mathrm{WI}$ and high-signal intensity on ASL images using commercial software (Synapse ${ }^{\circledR}$ 3D Vincent, Ver.4.0, Fujifilm, Tokyo, Japan) with semiautomatic drawing. Volumes of interest (VOIs) were placed only on the high signal intensity regions of $\mathrm{CE}+\mathrm{T} 1 \mathrm{WI}$ and ASL images (Fig. 1). Tumor position on CE+T1WI was used as the reference for VOI placement on ASL images. We did not include necrotic areas in tumors and avoided nontumoral tissues.
To reduce sampling errors due to inclusion of surrounding lowintensity regions within VOIs, we used cutoff values derived from VOIs encompassing contralateral healthy white matter. For both $\mathrm{CE}+\mathrm{T} 1 \mathrm{WI}$ and ASL, cutoff values were defined as the mean+ $2 \times$ standard deviation, which was subtracted from the VOI signal intensity histogram for each patient.

Next, we calculated the volume difference by subtracting the high-intensity volume obtained by ASL from the CE+T1WI volume (ASL tumor volume - CE tumor volume). We also calculated the volume ratio (ASL volume/CE volume) for each patient.

We investigated $\mathrm{CBF}$ parameters such as the absolute maximal tumor blood flow (TBF) and TBF ratio (normalized to blood flow in healthy white matter) between tumor groups using the Advantage Window AW 4.6 workstation (GE, Medical Systems, Milwaukee, WI). The regions of interest (ROI)'s were placed on maximal signal intensity areas of the tumor lesions on $\mathrm{CE}+\mathrm{T} 1 \mathrm{WI}$ and copied to corresponding ASL maps. All measurements were acquired independently by two neuroradiologists (M.G and M.H) blinded to the diagnostic information and averaged for analyses. Only values with intra-class correlation coefficients (ICC) $\geq 0.90(p<0.001)$ were included in the final analysis.

\section{STATISTICAL ANALYSIS}

All statistical analyses were performed using SPSS Ver. 20 (IBM, Armonk, NY, USA). The Mann-Whitney U test was used to assess differences in variables between GBM and metastasis. Receiver operating characteristic (ROC) curve analysis was used to assess diagnostic accuracy for differentiating GBM from metastasis. The level of significance was set at $\mathrm{p}<0.05$.

\section{RESULTS}

Both subtracted tumor volume (ASL-measured volume minus CE-T1WI-measured volume) and ratio of tumor volume (ASLmeasured volume/CE-T1WI-measured volume) differed significantly between the tumor types (Table 1 ), with subtracted tumor volume significantly larger for GBM than for metastasis $(p<0.001)$. Similarly, the volumetric ratio was significantly higher for GBM than for metastasis $(p<0.001)$. In contrast, differences in the TBF and $\mathrm{TBF}$ ratio did not reach statistical significance $(\mathrm{p}=0.85$ and $\mathrm{p}=$ 0.69 , respectively).

The areas under the ROC curves for subtracted tumor volume and tumor volume ratio were 0.865 and 0.852 , respectively (Fig. 2). Subtracted tumor volume $>2.7 \mathrm{ml}$ distinguished GBM from metastasis with $96 \%$ sensitivity and $76.9 \%$ specificity, and a ratio $>1.14$ distinguished GBM from metastasis with $96 \%$ sensitivity and $69.2 \%$ specificity. The AUC for TBF was 0.674 , with $60 \%$ sensitivity and $69.2 \%$ specificity. The AUC of the TBF ratio was 0.683 , with $56 \%$ sensitivity and $76.9 \%$ specificity. Diagnostic accuracy was significant for both tumor volume difference and tumor volume ratio $(\mathrm{P}<$ 0.001 for both) but not for TBF or TBF ratio.

\section{DISCUSSION}

The similar imaging manifestations of GBM and metastasis can complicate differential diagnosis, particularly when metastasis has a single mass. Survival time and treatment options differ substantially between GBM and metastasis. Median survival time is only 12.61 months for GBM and even shorter for metastasis, necessitating timely and accurate diagnosis. The main treatment for GBM is immediate removal of as much of the tumor as possible, followed by radiation and chemotherapy. Metastasis treatment 


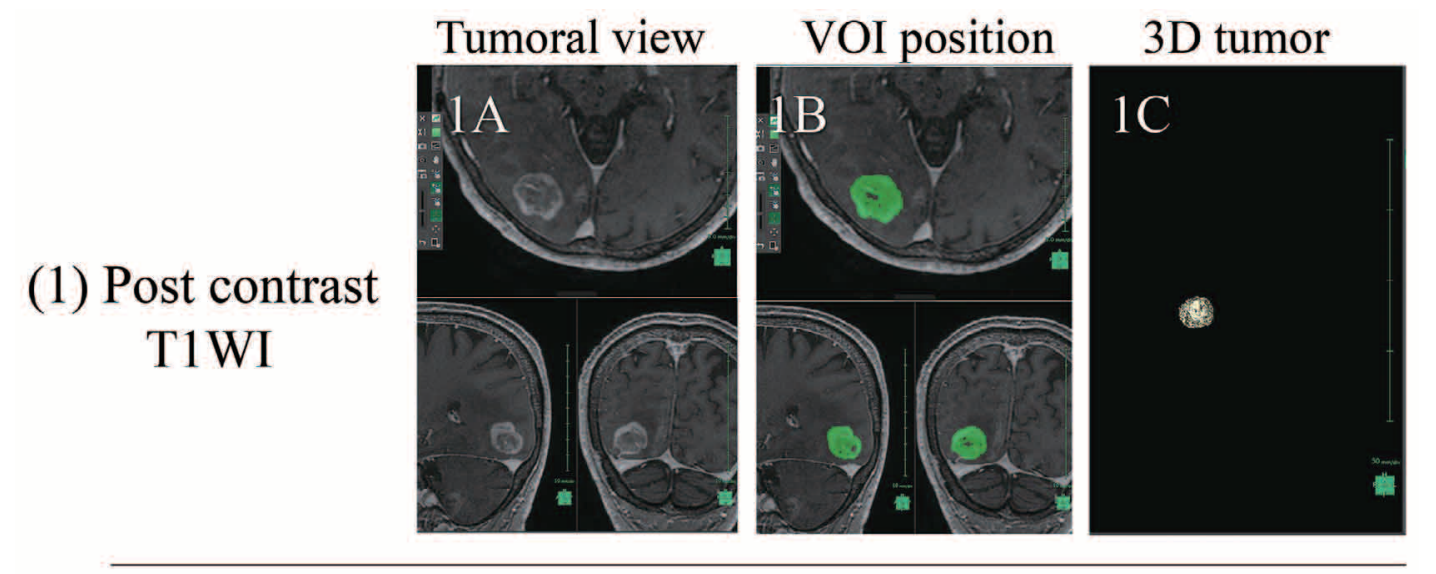

\section{(2) ASL}
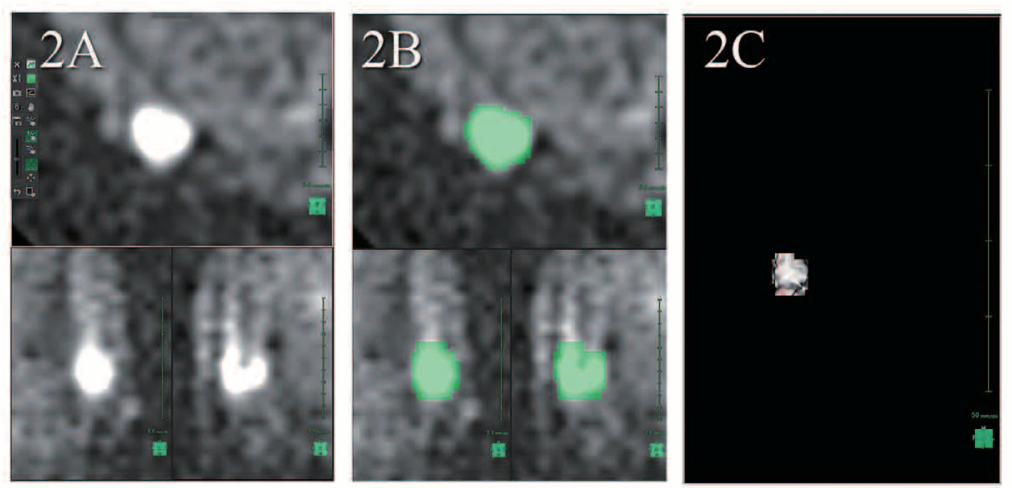

Fig. 1. An example volume of interest (VOI) drawn on an image of glioblastoma (GBM). (1A, 1B) Contrast-enhanced T1-weighted images (CE+ T1WI), (2A, 2B) ASL images (from left, axial, sagittal, and coronal planes) and (1C, 2C) 3D view of VOI. The tumor manifested as a contrast enhancement on CE+T1WI and a high signal intensity on ASL images. Tumor VOI (green) included only abnormal high signal intensity.

Table 1

\begin{tabular}{lccr}
\hline & GBM $(\mathrm{n}=25)$ & MET $(\mathrm{n}=13)$ & P value \\
\hline Sex (male\%) & 68 & 46.15 & 0.286 \\
Age & $67.36 \pm 10.79$ & $61.23 \pm 10.21$ & 0.970 \\
Volume difference $(\mathrm{ml})$ & $21.55 \pm 22.12$ & $3.49 \pm 11.59$ & $<0.001$ \\
Volume ratio & $3.2 \pm 2.4$ & $1.2 \pm 0.89$ & $<0.001$ \\
TBF $(\mathrm{ml} / 100 \mathrm{~g} / \mathrm{min})$ & $136.5 \pm 58.16$ & $107.6 \pm 64.93$ & 0.85 \\
TBF ratio & $5.66 \pm 2.35$ & $4.26 \pm 2.36$ & 0.69 \\
\hline
\end{tabular}

GBM, glioblastoma multiforme ; MET, metastasis ; TBF, tumor blood flow

options depend on whether there is a single or multiple lesion ; generally, for patients with a single lesion, surgery is considered effective, but a recent study concluded that whole brain radiation therapy combined with stereotactic radiosurgery could be better (23-25).

In the present study, comparative volumetric assessment using ASL and CE+T1WI differentiated GBM from metastasis with clinical significance. ROC analysis (Fig. 2) showed that both volume difference and volume ratio were higher for GBM compared with metastatic tumors. The area under the ROC curve was highest for subtracted tumor volume (0.865) and lowest for TBF (0.674), and both TBF and TBF ratio were less sensitive and specific indicators of tumor type than were volumetric parameters. In sum, a larger high-intensity signal volume on ASL compared with CE volume is suggestive of GBM, while a smaller volume difference suggests metastasis.

It is well known that ASL signal intensity is strongly correlated with tumor vascularity, allowing histopathological tumor diagnosis and grading $(9,10,26)$. The extensive tumor angiogenesis associated with GBM (neovascularization) results in vascular abnormalities such as microvessel proliferation, endothelial hyperplasia, vessel dilation, and high vessel tortuosity. GBM generally grows diffusely into normal brain tissue due to the rich vascular network created $(14,24,27)$. In contrast, vascularity of metastasis is mostly resembled the vessels of primary site tumors and microvessel density is only higher in intratumoral lesion. It is observed that angiogenesis in metastasis is higher than in normal tissue but metastasis is much less vascular. While angiogenesis is higher than in normal tissue, angiogenic proliferation is very low in the peritumoral region $(14,15)$. Therefore, several studies have found 


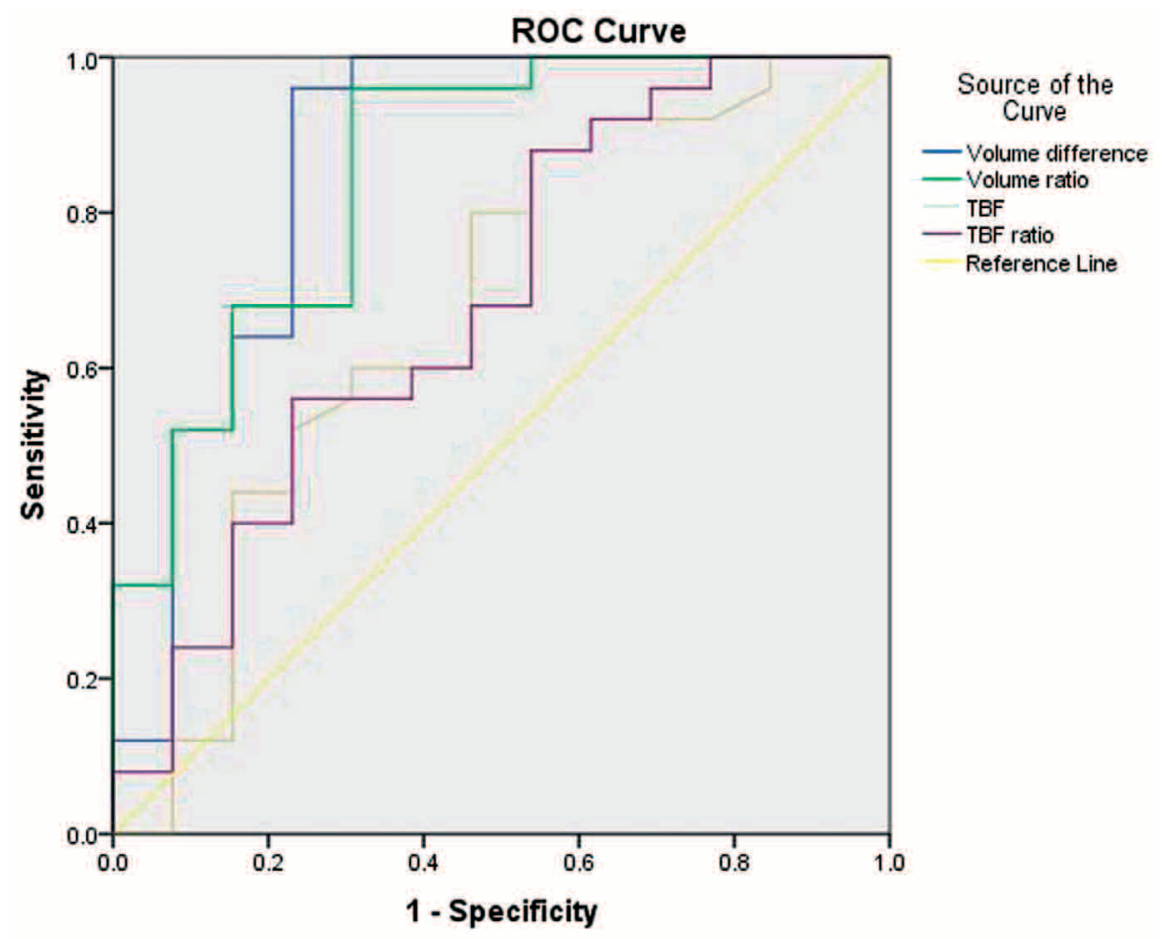

Diagonal segments are produced by ties.

Fig. 2. Receiver operating characteristic (ROC) curves for determining the discrimination accuracy of the variables. The volume difference and volume ratio showed greater sensitivity and specificity for distinguishing GBM from metastasis than absolute maximal tumor blood flow (TBF) and TBF ratio as indicated by areas under the ROC curves.
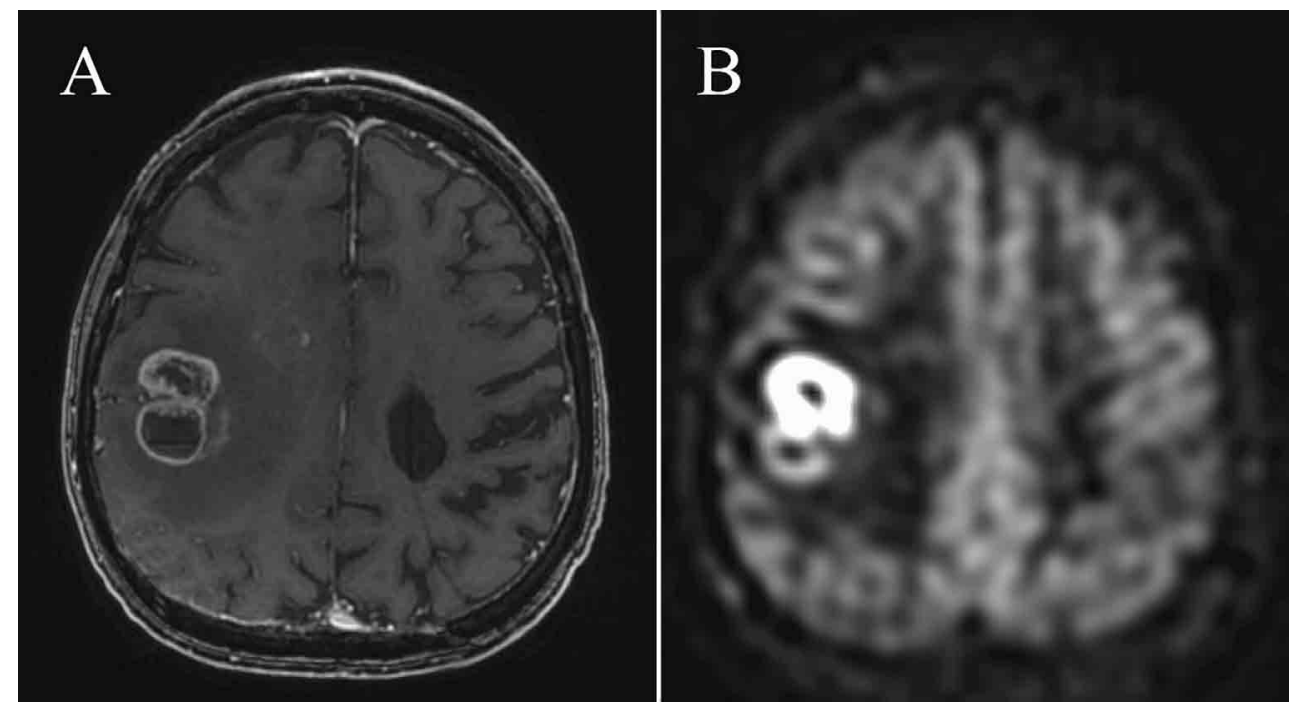

Fig. 3. Glioblastoma with surrounding edema (WHO grade IV) in a 75-year-old male patient. (A) A CE+T1WI showing a solid contrast-enhanced lesion. (B) The tumor exhibits a large hyperintensity on ASL images.

associations of intratumoral vascular shunts with tumor growth, particularly for GBM $(12,28)$. Neovascularization related with tumor development can lead to blood-brain barrier disruption, which produces leaky vessels with stagnant flow (29). Both of vascular shunts with rapid tracer transit and stagnant blood flow would give high signal intensity on ASL images $(11,12,30)$.

On the other hand, a difference in tumoral edema can affect tracer transit time in ASL. It is found that a higher degree of surrounding edema was more likely in metastasis, while GBM produced greater contrast enhancement. Cerebral edema in patients with metastatic neoplasms is often vasogenic due to vascular permeability, whereas GBM often exhibits a combination of cytotoxic edema and cell infiltration $(4,5,14)$. Due to these differences in angiogenesis, we suspect that a difference in ASL signal stemming from the tumor 

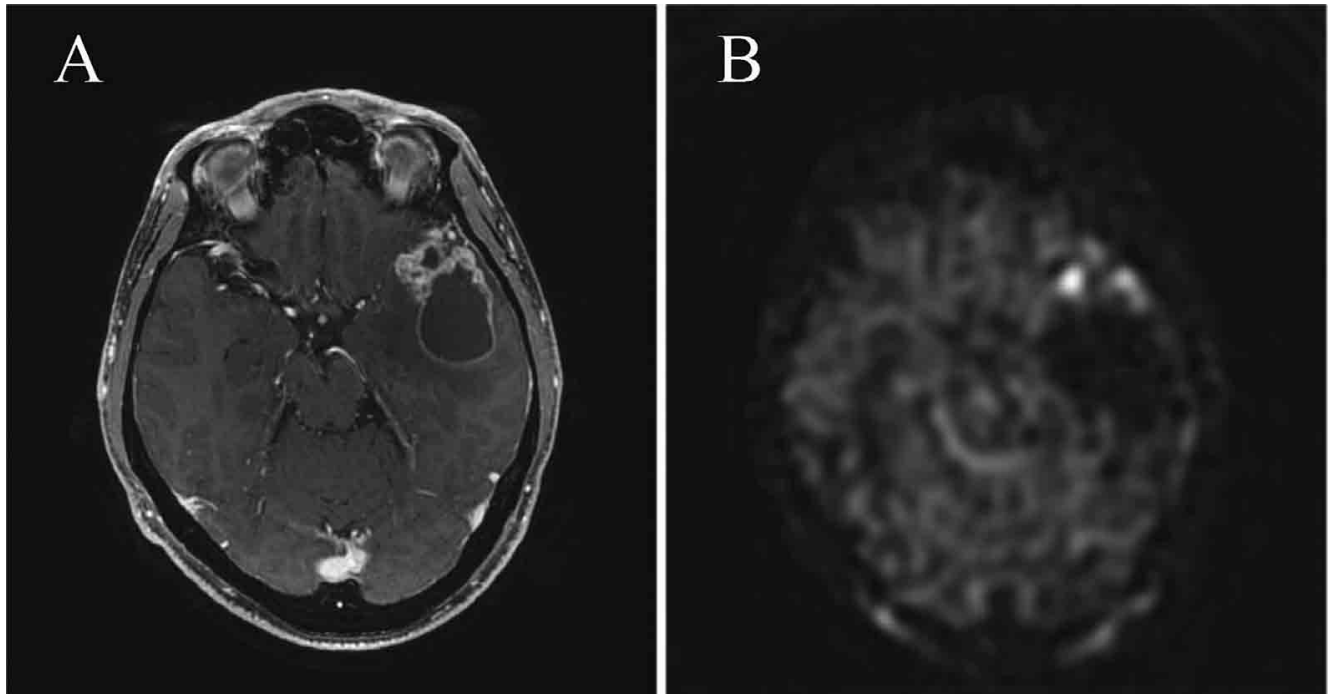

Fig. 4. A histologically confirmed metastatic tumor from lung cancer in a 71-year-old female patient. (A) Ring enhanced solid lesion on CE+T1WI. (B) The tumor shows hyperperfusion on ASL, but the area of high signal intensity is small compared with the contrast intensity.

itself is unlikely between GBM and MET.

Cerebral blood flow is considered the most reliable measurement parameter of ASL for clinical evaluation (9). In our study, GBM exhibited a slightly higher TBF than metastasis, but neither the difference of TBF and TBF ratio reached the level of statistical significance (Table 1). Previous studies of contrast perfusion MRI found no significant difference in TBF between malignant glioma and metastasis (3-5), consistent with our results. It is noted that both GBM and metastasis are highly vascular malignant tumors, and specific types of metastasis such as melanoma and renal carcinoma may show markedly elevated $\operatorname{TBF}(3,6)$. In the present study, a metastatic tumor from colon carcinoma showed the highest perfusion value by ASL. Alternatively, several studies have reported higher perfusion for malignant glioma than for metastasis (6), and greater perfusion in the peritumoral region is considered sufficient to differentiate malignant glial tumors $(4,5)$. However, we did not focus on peritumoral regions as these have been examined in many previous comparative studies on GBM and metastasis. Nevertheless, the major portion of GBM lesions show surrounding edema (7), while some types of metastases show very high perfusion $(3,6,14)$. It thus appears difficult to differentiate metastasis from GBM by differences in ASL-measured TBF.

Multiple volumetric parameters derived from conventional MRI have been investigated for brain tumor evaluation, most assessing tumor treatment response rather than differentiation of tumor type. In this study, we used 3D volumetry of ASL, a novel perfusion method for assessing various cerebral diseases (9). According to our findings, the combination of volumes of ASL high signal and contrast enhancement may be useful for differentiating GBM from metastasis, particularly as the use of ASL is increasing due to other non-contrast benefits. In addition to diagnostic applications, volumetric analysis of ASL signal changes could be used for physiological measurement of microvascular changes, which could be complemented by traditional sequences for assessment of morphological changes.

Limitations of the study include a relatively small number of tumor cases, in particular metastasis cases. We also did not consider tumor size, and it is possible that larger metastatic tumors show more expansive ASL high signal volumes than smaller GBMs do. The acquisition time of this volumetry method may be longer than conventional MRI studies due to the need for multiple 3D imaging datasets (ASL and CE sequences). Furthermore, this method may be inappropriate for subjects with low signal intensity lesions on both imaging modalities.

\section{CONCLUSION}

We demonstrated that the larger volume of tumoral high signal intensity on ASL images compared with conventional CE+T1WI can differentiate GBM from metastasis with relatively high sensitivity and specificity, whereas ASL-derived TBF cannot. Differences in lesion volume between GBM and metastasis were much larger on ASL images than conventional CE+T1WI images.

\section{CONFLICT OF INTEREST STATEMENT}

None

\section{REFERENCES}

1. Davis FG, Freels S, Grutsch J, Barlas S, Brem S : Survival rates in patients with primary malignant brain tumors stratified by patient age and tumor histological type : an analysis based on Surveillance, Epidemiology, and End Results (SEER) data, 1973-1991. J Neurosurg 88:1-10, 1998

2. Gavrilovic IT, Posner JB : Brain metastases : epidemiology and pathophysiology. J Neurooncol 75 : 5-14, 2005

3. Hakyemez B, Erdogan C, Bolca N, Yildirim N, Gokalp G, Parlak M : Evaluation of different cerebral mass lesions by perfusion-weighted MR imaging. J Magn Reson Imaging 24 : 817-24, 2006

4. Tsougos I, Svolos P, Kousi E, Fountas K, Theodorou K, Fezoulidis I, Kapsalaki E : Differentiation of glioblastoma multiforme from metastatic brain tumor using proton magnetic resonance spectroscopy, diffusion and perfusion metrics at 3 T. Cancer Imaging $12: 423-36,2012$

5. Law M, Cha S, Knopp EA, Johnson G, Arnett J, Litt AW : Highgrade gliomas and solitary metastases : differentiation by using perfusion and proton spectroscopic MR imaging. Radiology 
$222: 715-21,2002$

6. Kremer S, Grand S, Berger F, Hoffmann D, Pasquier B, Remy C, Benabid AL, Le Bas JF : Dynamic contrast-enhanced MRI : differentiating melanoma and renal carcinoma metastases from high-grade astrocytomas and other metastases. Neuroradiology $45: 44-9,2003$

7. Schoenegger K, Oberndorfer S, Wuschitz B, Struhal W, Hainfellner J, Prayer D, Heinzl H, Lahrmann H, Marosi C, Grisold W : Peritumoral edema on MRI at initial diagnosis : an independent prognostic factor for glioblastoma? Eur J Neurol $16: 874-8,2009$

8. Detre JA, Alsop DC : Perfusion magnetic resonance imaging with continuous arterial spin labeling: methods and clinical applications in the central nervous system. Eur J Radiol 30 : 115-24, 1999

9. Detre JA, Rao H, Wang DJ, Chen YF, Wang Z : Applications of arterial spin labeled MRI in the brain. J Magn Reson Imaging 35 : 1026-37, 2012

10. Noguchi T, Yoshiura T, Hiwatashi A, Togao O, Yamashita K, Nagao E, Shono T, Mizoguchi M, Nagata S, Sasaki T, Suzuki SO, Iwaki T, Kobayashi K, Mihara F, Honda H : Perfusion imaging of brain tumors using arterial spin-labeling : correlation with histopathologic vascular density. AJNR Am J Neuroradiol $29: 688-93,2008$

11. Le TT, Fischbein NJ, Andre JB, Wijman C, Rosenberg J, Zaharchuk G : Identification of venous signal on arterial spin labeling improves diagnosis of dural arteriovenous fistulas and small arteriovenous malformations. AJNR Am J Neuroradiol $33: 61-8,2012$

12. Wolf RL, Wang J, Detre JA, Zager EL, Hurst RW : Arteriovenous shunt visualization in arteriovenous malformations with arterial spin-labeling MR imaging. AJNR Am J Neuroradiol $29: 681-7,2008$

13. Abe T, Mizobuchi Y, Sako W, Irahara S, Otomi Y, Obama Y, Nakajima K, Khashbat D, Majigsuren M, Kageji T, Nagahiro S, Harada M : Clinical Significance of Discrepancy between Arterial Spin Labeling Images and Contrast-enhanced Images in the Diagnosis of Brain Tumors. Magn Reson Med Sci $14: 313-9,2015$

14. Cha S, Lupo JM, Chen MH, Lamborn KR, McDermott MW, Berger MS, Nelson SJ, Dillon WP : Differentiation of glioblastoma multiforme and single brain metastasis by peak height and percentage of signal intensity recovery derived from dynamic susceptibility-weighted contrast-enhanced perfusion MR imaging. AJNR Am J Neuroradiol 28 : 1078-84, 2007

15. Bugyik E, Dezso K, Reiniger L, Laszlo V, Tovari J, Timar J, Nagy P, Klepetko W, Dome B, Paku S : Lack of angiogenesis in experimental brain metastases. J Neuropathol Exp Neurol 70 : 979-91, 2011

16. Joe BN, Fukui MB, Meltzer CC, Huang QS, Day RS, Greer PJ, Bozik ME : Brain tumor volume measurement : comparison of manual and semiautomated methods. Radiology 212 : 811 6,1999
17. Wang MY, Cheng JL, Han YH, Li YL, Dai JP, Shi DP : Measurement of tumor size in adult glioblastoma : classical crosssectional criteria on 2D MRI or volumetric criteria on high resolution 3D MRI? Eur J Radiol 81 : 2370-4, 2012

18. Ellingson BM, Malkin MG, Rand SD, LaViolette PS, Connelly JM, Mueller WM, Shmainda KM : Volumetric analysis of functional diffusion maps is a predictive imaging biomarker for cytotoxic and anti-angiogenic treatments in malignant gliomas. J Neurooncol 102 : 95-103, 2011

19. Georgiadis P, Cavouras D, Kalatzis I, Glotsos D, Athanasiadis E, Kostopoulos S, Sifaki K, Malamas M, Nikiforidis G, Solomou $\mathrm{E}$ : Enhancing the discrimination accuracy between metastases, gliomas and meningiomas on brain MRI by volumetric textural features and ensemble pattern recognition methods. Magn Reson Imaging $27: 120-130,2009$

20. Louis DN, Ohgaki H, Wiestler OD, Cavenee WK, Burger PC, Jouvet A, Sheithauer BW, Kleiheus P : The 2007 WHO Classification of Tumours of the Central Nervous System. Acta Neuropthol 114 : 97-109, 2007

21. Osborn AG, Salzman KL, Barkovich AJ : Diagnostic Imaging Brain. In : Osborn AG eds. Section 6 Neoplasms. Amirsys Publishing Inc, Canada, 2009. pp. I-6-2-182

22. Dai W, Garcia D, de Bazelaire C, Alsop DC : Continuous flowdriven inversion for arterial spin labeling using pulsed radio frequency and gradient fields. Magn Reson Med 60 : 148897, 2008

23. Johnson DR, Ma DJ, Buckner JC, Hammack JE : Conditional probability of long-term survival in glioblastoma : a population-based analysis. Cancer $118: 5608-13,2012$

24. Omuro A, DeAngelis LM : Glioblastoma and other malignant gliomas : a clinical review. JAMA 310 : 1842-50, 2013

25. Jenkinson MD, Haylock B, Shenoy A, Husband D, Javadpour $\mathrm{M}$ : Management of cerebral metastasis : evidence-based approach for surgery, stereotactic radiosurgery and radiotherapy. Eur J Cancer 47 : 649-55, 2011

26. Cebeci H, Aydin O, Ozturk-Isik E, Gumus C, Inecikli F, Bekar A, Kocaeli H, Hakyemez B : Assesment of perfusion in glial tumors with arterial spin labeling ; comparison with dynamic susceptibility contrast method. Eur J Radiol 83 : 1914-9, 2014

27. Jain RK, di Tomaso E, Duda DG, Loeffler JS, Sorensen AG, Batchelor TT : Angiogenesis in brain tumours. Nat Rev Neurosci $8: 610-22,2007$

28. Mariani L, Schroth G, Wielepp JP, Haldemann A, Seiler RW : Intratumoral arteriovenous shunting in malignant gliomas. Neurosurgery $48: 353-7$; discussion 7-8, 2001

29. Krakstad C, Chekenya M : Survival signalling and apoptosis resistance in glioblastomas : opportunities for targeted therapeutics. Mol Cancer $9: 135,2010$

30. Zaharchuk G, Bammer R, Straka M, Shankaranarayan A, Alsop DC, Fischbein NJ, Atlas SW, Moseley ME : Arterial spin-label imaging in patients with normal bolus perfusion-weighted MR imaging findings : pilot identification of the borderzone sign. Radiology 252 : 797-807, 2009 